\title{
COMPUTATIONAL ANALYSIS OF INSERTED POROUS BLOCKS INTO HORIZONTAL CONCENTRIC ANNULI IN MIXED CONVECTION MODE
}

\author{
Y. Ould-Amer ${ }^{1, *}$
}

\begin{abstract}
This study numerically investigates the effect of porous blocks thickness on laminar mixed convection in a horizontal annulus. With attached four porous blocks on the inner cylinder, steady 3D laminar mixed convection is presented for the fully developed region of horizontal concentric annuli.

Results are presented for two values of porous blocks thickness and a range of the values of the Grashoff number, Darcy number and the conductivity ratio between the porous medium and the fluid. Results are presented in the form of contours plots of the streamlines and for the temperature isotherms, and in terms of the overall heat transfer coefficients and friction factor.

The average Nusselt number increases significantly with an increase of the thickness of porous blocks. With the use of the four porous blocks, the friction factor is consequently increased compared with the situation without porous blocks. The decrease of the Darcy number leads to an increase of the friction factor.

If the fully fluid case is taken as a reference, the use of porous blocks is justified only when the ratio of the average Nusselt number to the friction factor is enhanced.
\end{abstract}

\section{Keywords: Renewable Mixed Convection, Concentric Annuli, Porous, Thickness}

\section{INTRODUCTION}

Fluid flow and heat transfer in annular passages are important in many engineering applications such as refrigeration, chemical process, cooling of turbine rotors, thermal storage systems, food industries, electrical transport and nuclear energy production.

Double pipe heat exchangers in chemical process, gas - cooled electrical cables, double pipe heat exchangers and the cooling of nuclear fuel rods represent industrial examples in which annular flow heat transfer is encountered. In these situations, the forced convection in the duct flow is often significantly influenced by body forces, which tend to produce secondary flows in the duct cross section. The main focus of these systems is to enhance the overall heat transfer coefficient.

Laminar mixed convection between concentric horizontal cylinders is presented in [1-4]. Niekele and Patankar [4] have examined numerically the effect of buoyancy forces on heat transfer in an annulus. Results are presented for a range of the values of the Gashoff number, the Prandtl number, and the radius ratio of the annulus. Habib and Negm [5] studied numerically the laminar mixed convection in the fully region of horizontal concentric annuli for the case of the non-uniform circumferential heating. The problem of mixed convection in a horizontal annulus with isothermal walls, the inner heated and the outer cooled, was studied numerically by Muralidhar [6].

In the last decades, the use of porous material to improve the heat transfer is of topical interest. Field applications of heat transfer in porous media can be found in [7]. Vanover and Kulacki [8] conducted experiments in a porous annulus with the inner cylinder heated by constant heat flux and the outer cylinder isothermally cooled. The medium consisted of 1- and 3-mm glass beads saturated with water. They found that when the Rayleigh number is large the values of the Nusselt number for mixed convection may be lower than the free convection values. They attributed this to a restructuring of the flow as forced convection begins to play a dominant role.

Laminar mixed convection in the thermal entry region of the concentric horizontal annulus of radius 2 has been experimentally studied by Mohammed et al. [9]. The experimental setup consists of a stainless annulus with inner tube subjected to a constant wall heat flux boundary condition and an adiabatic outer annulus. Their experimental results show that the free convection tends to decrease the heat transfer at low Reynolds number while to increase the heat transfer for high Reynolds number.

This paper was recommended for publication in revised form by Regional Editor Jaap Hoffman Hoffman

${ }^{1}$ USTHB, Faculty of Mechanical and Process Engineering (FGMGP), Laboratory of Multiphase Transport and Porous Media

(LTPMP), Département Energétique, B.P. 32 El-Alia Bab Ezzouar 16111 Algiers, Algeria

${ }^{*} E$-mail address: youldamer@usthb.dz

Manuscript Received 22 January 2016, Accepted 1 July 2016 
Ould-Amer [10] carried out numerical analysis to investigate the performance of an innovative thermal system to improve the heat transfer in the horizontal annulus. With attached four porous blocks on the inner cylinder, steady laminar mixed convection is examined for the fully developed region of horizontal concentric annuli. Their results show the significant improve in heat transfer with highly conductive porous medium; however, the friction factor is increased compared with situations without porous blocks.

Venugopal et al. [11] studied experimentally the mixed convection in vertical duct filled with metallic porous structure. The main focus of their work is to examine the potential of a porous insert for augmenting heat transfer from the heated wall of vertical duct under forced flow conditions.

The objective of this paper is to evaluate the effect of a porous blocks thickness on mixed convection, on the flow and heat transfer in the horizontal annulus. Four porous blocks are fixed on the inner cylinder. The analysis is performed for laminar flow and for thermally and hydrodynamically developed conditions. This work is an extension of the previous study of Ould-Amer [10, 12-13], indeed, the effect of the thickness of porous blocks have not been studied in the previous works.

\section{ANALYSIS}

The physical problem considered in the present paper is shown in Figure 1. Four porous blocks are fixed on the outside of the inner cylinder. The porous blocks are considered homogeneous, isotropic and saturated by an incompressible fluid. The outer cylinder is insulated while the inner cylinder has circumferentially uniform surface temperature and axially uniform heat transfer rate. In the fully developed region of the flow, the velocity components $\mathrm{u}, \mathrm{v}$ and $\mathrm{w}$ in the $\varphi, \mathrm{r}$ and $\mathrm{z}$ coordinates become independent of the axial distance $\mathrm{z}$. The density of the fluid is considered constant except in the buoyancy term (Boussinesq approximation). A local thermal equilibrium takes place between the fluid and the porous medium. Because of the symmetry about the vertical center line shown in Fig. 1, the analysis is confined to a right half of the annulus.

The continuity, energy and momentum equations that govern the physical situation can be written in dimensionless form as follows [4,7]:

$$
\begin{gathered}
\frac{\partial U}{\partial \varphi}+\frac{\partial(\eta V)}{\partial \eta}=0 \\
\mathrm{~V} \frac{\partial \theta}{\partial \eta}+\frac{\mathrm{U}}{\eta} \frac{\partial \theta}{\partial \varphi}+\frac{4 \frac{W}{W}}{\operatorname{Pr}(1+R R)}=\frac{1}{\operatorname{Pr}}\left[\frac{1}{\eta} \frac{\partial}{\partial \eta}\left(\mathrm{R}_{\mathrm{C}} \eta \frac{\partial \theta}{\partial \eta}\right)+\frac{1}{\eta^{2}} \frac{\partial}{\partial \varphi}\left(\mathrm{R}_{\mathrm{C}} \frac{\partial \theta}{\partial \varphi}\right)\right] \\
\frac{1}{\varepsilon^{2}}\left[V \frac{\partial V}{\partial \eta}+\frac{\mathrm{U}}{\eta} \frac{\partial V}{\partial \varphi}-\frac{U^{2}}{\eta}\right]=-\frac{\partial P}{\partial \eta}-\operatorname{Gr} \theta \cos \varphi+R_{V}\left[\frac{\partial}{\partial \eta}\left(\eta \frac{\partial V}{\partial \eta}\right)+\frac{1}{\eta^{2}} \frac{\partial^{2} V}{\partial \varphi^{2}}-\frac{V}{\eta^{2}}-\frac{2}{\eta^{2}} \frac{\partial U}{\partial \varphi}\right] \\
\left.\frac{1}{\varepsilon^{2}}\left[V \frac{\partial U}{\partial \eta}+\frac{\mathrm{U}}{\eta} \frac{\partial U}{\partial \varphi}+\frac{U V}{\eta}\right]=-\frac{1}{\sqrt{D a}} \mid \frac{\partial P}{\partial \varphi}+\operatorname{Vin}\right] \\
-\lambda\left[\frac{U}{D a}+\frac{C_{F}}{\sqrt{D a}}|\vec{V}| U\right] \\
\frac{1}{\varepsilon^{2}}\left[V \frac{\partial W}{\partial \eta}+\frac{\mathrm{U}}{\eta} \frac{\partial W}{\partial \varphi}\right]=1+R_{V}\left[\frac{1}{\eta} \frac{\partial}{\partial \eta}\left(\eta \frac{\partial W}{\partial \eta}\right)+\frac{1}{\eta^{2}} \frac{\partial^{2} W}{\partial \varphi^{2}}\right]-\lambda \frac{W}{D a}
\end{gathered}
$$

With dimensionless boundary conditions:

$$
\begin{array}{lll}
\eta=\eta_{i} & U=V=W=0 & \theta=0 \\
\eta=\eta_{o} & U=V=W=0 & \frac{\partial \theta}{\partial \eta}=0
\end{array}
$$




$$
\varphi=0, \pi \quad U=0 \quad \frac{\partial V}{\partial \varphi}=\frac{\partial W}{\partial \varphi}=\frac{\partial \theta}{\partial \varphi}=0
$$

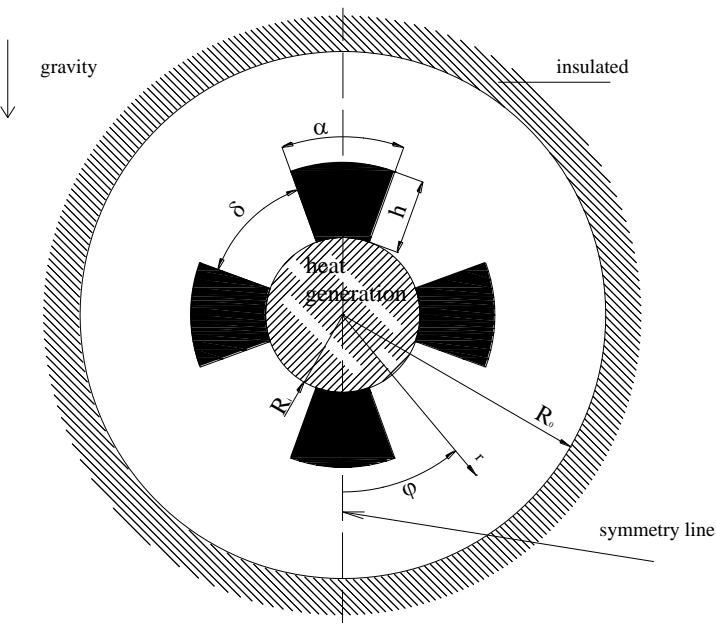

Figure 1. Physical model

For convenience, the pressure is redefined as explained in the appendix. In the energy equation, the relation between axial temperature gradient and the average heat flux at the surface of the inner cylinder is given in the appendix. At the interfaces which separate the fully fluid zone and the porous blocks, the continuity of flow, pressure, temperature, stress and also energy are adopted. The porosity is set equal to one in the fully fluid zone and the $\lambda$ parameter and the conductivity ratio $\mathrm{R}_{\mathrm{C}}$ are defined as follows:

$$
\begin{gathered}
\lambda= \begin{cases}0 & \text { in thefully fluid zone } \\
1 & \text { in the porous bloks }\end{cases} \\
\mathrm{R}_{\mathrm{C}}= \begin{cases}1 \text { in thefully fluid zone } \\
\mathrm{k}_{\mathrm{P}} / \mathrm{k}_{\mathrm{f}} & \text { in the porousbloks }\end{cases}
\end{gathered}
$$

\section{NUMERICAL PROCEDURE}

The set of differential equations governing mixed convection in the horizontal concentric annuli is transformed into a system of algebraic equations with the use of the control volume approach. The SIMPLER algorithm is used for the calculation of the flow field and temperature. The system of algebraic equations is solved iteratively by means of the Thomas algorithm. Under-relaxation factors are introduced to avoid the divergence of the strongly non-linear system. A nonuniform staggered grid of $74 \times 64$ nodes, over the entire domain, was selected on the basis of a grid sensitivity study presented in Table 1 . Convergence is controlled in terms of the relative error for the variables $U, V, \mathrm{~W}, P, \theta$ and the mass residual in each control volume. The convergence criterion is:

$$
\left|\frac{\Phi_{i j}^{n+1}-\Phi_{i j}^{n}}{\Phi_{i j}^{n}}\right|<10^{-5} \text { and } \mid \text { residualmass }\left.\right|_{\text {in eachcontrol volume }}<10^{-5}
$$

With $\Phi$ corresponding to $\mathrm{U}, \mathrm{V}, \mathrm{W}, \mathrm{P}$ or $\theta$, and $n$ and $n+1$ indicating two consecutive iterations. The discontinuity between the porous blocks and fully fluid zone is handled with the use of the harmonic mean formulation suggested by [14]. The porosity discontinuity at the interface between purely fluid zone and porous blocks is evaluated by linear interpolation. The present code was validated by comparing the results obtained with our code for the fully fluid case, with those of Nieckele and Patankar [4]. Good agreement between the results is 
Table 1.Grid sensitivity for $\mathrm{Gr}=10^{6}, \mathrm{Da}=10^{-3}, \mathrm{Pr}=0.7, \mathrm{C}_{\mathrm{F}}=0.011, \varepsilon=0.9, \alpha=60^{\circ}$, $\mathrm{R}_{\mathrm{C}}=50, \mathrm{RR}=2, \mathrm{H}=0.25$

\begin{tabular}{|c|c|c|}
\hline $\begin{array}{c}\text { Grid } \boldsymbol{\varphi} \\
\times \eta\end{array}$ & $\mathrm{Nu}_{\mathrm{b}}$ & $f \mathrm{Re}$ \\
\hline $42 \times 32$ & 26.128 & 301.160 \\
\hline $50 \times 42$ & 26.099 & 300.917 \\
\hline $74 \times 64$ & 26.052 & 300.902 \\
\hline $106 \times 92$ & 26.043 & 300.984 \\
\hline
\end{tabular}

Table 2.Comparison of present results with those of Nieckele and Patankar [4] for $\operatorname{Pr}=0.7, \mathrm{RR}=2$

\begin{tabular}{|c|c|c|c|c|}
\hline \multirow{2}{*}{ Gr } & \multicolumn{2}{|c|}{ Present code } & \multicolumn{2}{c|}{ [4] } \\
\cline { 2 - 5 } & $\frac{f \mathrm{Re}}{(f \mathrm{Re})_{0}}$ & $\frac{\mathrm{Nu}_{\mathrm{b}}}{\overline{\mathrm{Nu}_{0}}}$ & $\frac{f \mathrm{Re}}{(f \mathrm{Re})_{0}}$ & $\frac{\mathrm{Nu}_{\mathrm{b}}}{\overline{\mathrm{Nu}_{0}}}$ \\
\hline $10^{3}$ & 1.0000 & 1.0000 & 1.0000 & 1.0000 \\
\hline $10^{4}$ & 1.0002 & 1.0027 & 1.0000 & 1.0000 \\
\hline $10^{5}$ & 1.0115 & 1.1156 & 1.0083 & 1.1250 \\
\hline $10^{6}$ & 1.0793 & 1.4650 & 1.0833 & 1.4500 \\
\hline $10^{7}$ & 1.2692 & 1.7859 & 1.2667 & 1.8000 \\
\hline
\end{tabular}

obtained (Table 2). The situation of fully fluid case, i.e. without porous blocks, is obtained by setting the Darcy number equal to a large value (typically infinite) and the porosity, $\mathrm{R}_{\mathrm{V}}$ and $\mathrm{R}_{\mathrm{C}}$ to one.

\section{RESULTS AND DISCUSSION}

The numerical solutions are obtained for $\varepsilon=0.9, \mathrm{C}_{\mathrm{F}}=0.011, \operatorname{Pr}=0.7, \mathrm{R}_{\mathrm{V}}=1, \mathrm{RR}=2, \quad \alpha=60^{\circ}$, $\delta=30^{\circ}$. The others parameters are varied as follows: the Grashoff number from 10 to $10^{7}$, the Darcy number from $10^{-5}$ to $10^{-3}$ and the conductivity ratio from 1 to 50 . Two values for the porous blocks thickness are considered $(\mathrm{H}=0.25, \mathrm{H}=0.375)$. In this section, the flow structure and the heat transfer will be analyzed and discussed. The results include some local details of the flow and temperature fields and the overall heat transfer coefficient and friction factor.

\section{Streamlines and isotherms}

The secondary flow pattern and isotherms are displayed in Figures 2 and 3. Because of the symmetry about the vertical diameter, the isotherms are plotted in the left half and the streamlines in the right half. The value of $\psi$ listed for each figure is a measure of the strength of the secondary flow; $\psi$ is the maximum value of the dimensionless stream function.

At $\mathrm{H}=0.25$, and for all values of $\mathrm{Da}$ when $\mathrm{Gr} \leq 10^{5}$, the secondary flow forms a symmetrical eddy rotating in the clockwise direction, for instance figure $2 \mathrm{a}$. The isotherms are nearly circular with a deformation between two consecutive blocks. For $\mathrm{H}=0.375$ and all values of $\mathrm{Da}$, when $\mathrm{R}_{\mathrm{C}} \leq \mathrm{R}_{\text {Cthreshold }}$ and $G r \leq 10^{5}$, three

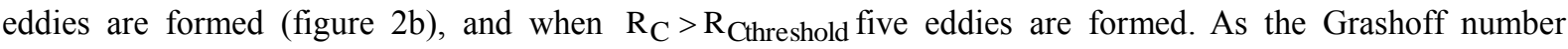
increases, the secondary motion becomes stronger as evidenced by the higher values of $\psi$, in this situation, two or three eddies are formed depending on the thickness of porous blocks (figures $3 \mathrm{a}$ and $3 \mathrm{~b}$ ). At higher values of $\mathrm{Gr}$, the isotherm pattern shows the thermal boundary layer near the porous blocks and between them close to heated inner duct. The presence of the porous blocks causes the distortion of the isotherms between two consecutive porous blocks, in this region the heat transfer decreases. The isotherms plots show that the thermal boundary layer along the surface of the two lower porous blocks get thinner as $\mathrm{H}$ goes from 0.25 to 0.375 , indicating improved heat transfer. However, in the upper region of the cross section of the horizontal annulus, the non-uniformities of temperatures occur, this behavior lead to the decrease of heat transfer in this zone. The presence of the porous blocks causes a supplementary resistance to the fluid flow as evidenced by the deformation of 
streamlines near the porous blocks when $\mathrm{H}$ goes from 0.25 to 0.375 . That is why the intensity of the secondary flow decreases when the thickness of the porous blocks is larger. Indeed, the secondary flow is decelerated causing

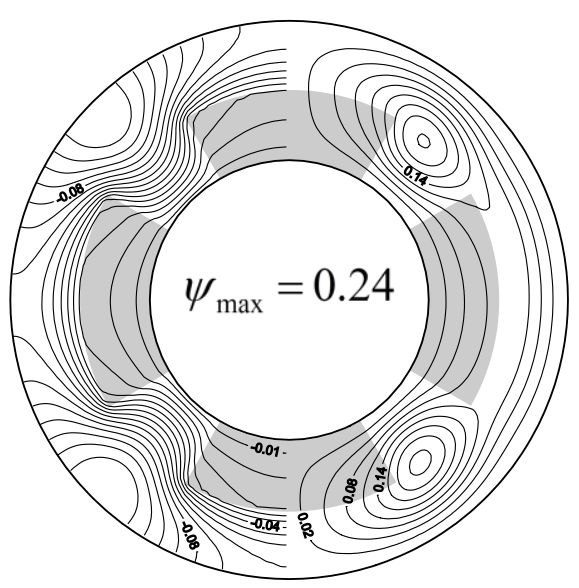

(a) $\mathrm{H}=0.25$

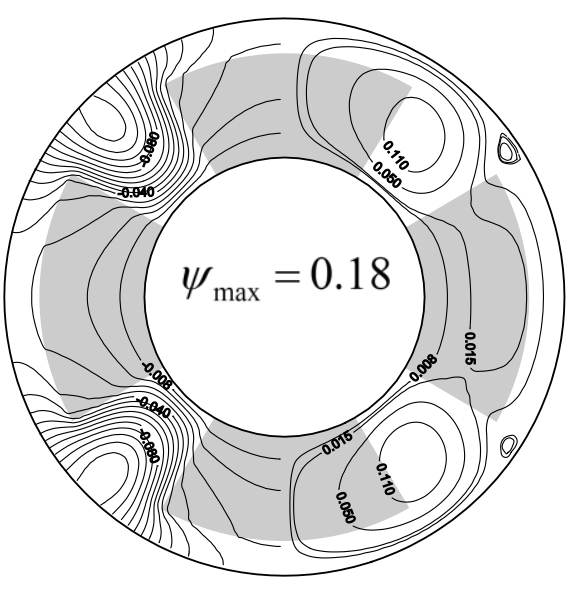

(b) $\mathrm{H}=0.375$

Figure 2. Isotherms and streamlines for $\mathrm{Da}=10^{-3}, \mathrm{Gr}=10^{4}, \mathrm{R}_{\mathrm{C}}=10$

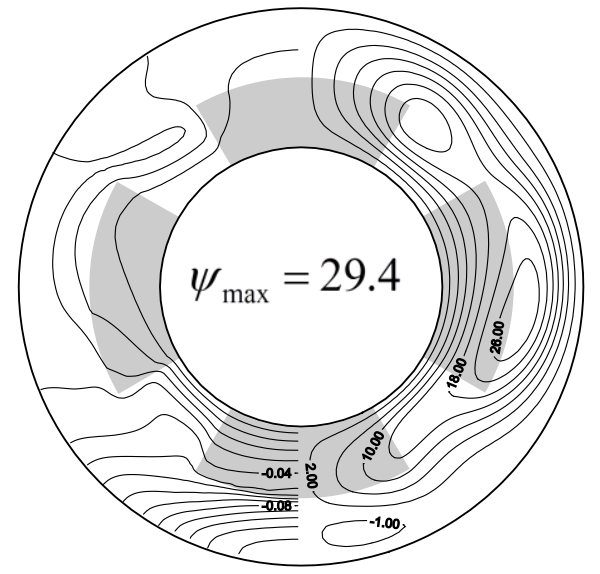

(a) $\mathrm{H}=0.25$

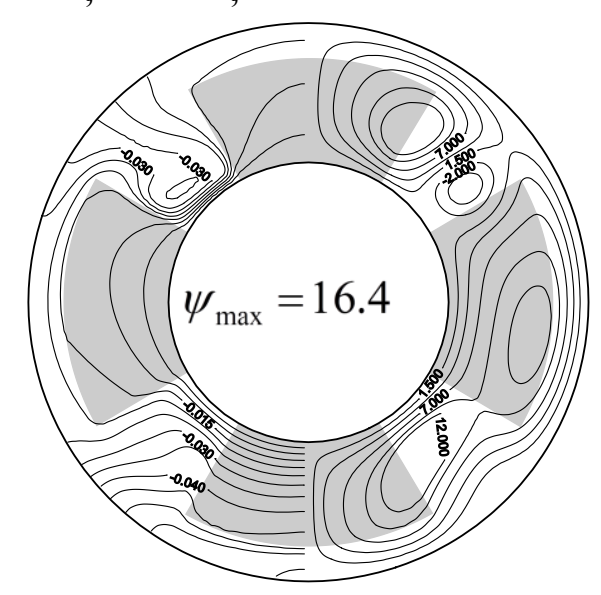

(b) $\mathrm{H}=0.375$

Figure 3. Isotherms and streamlines for $\mathrm{Da}=10^{-3}, \mathrm{G} r=10^{7}, \mathrm{R}_{\mathrm{C}}=10$

a significant increase in the flow resistance, the contribution of the secondary flow in heat transfer is thereby reduced.

\section{Overall heat transfer coefficient}

For the design purposes, the overall heat transfer in the device is of primary interest. The overall heat transfer results are presented in the form of the average Nusselt number $\mathrm{Nu}_{\mathrm{b}}$. The average Nusselt number is evaluated as:

$$
\mathrm{Nu}_{\mathrm{b}}=-\frac{1}{\theta_{\mathrm{b}}}
$$

Where $\theta_{\mathrm{b}}$ is the dimensionless bulk temperature of the fluid. 
The variation of $\mathrm{Nu}_{\mathrm{b}}$ with $\mathrm{Gr}$ is shown in Figures $4 \mathrm{a}$ and $4 \mathrm{~b}$. The influence of buoyancy is to increase $\mathrm{Nu}_{\mathrm{b}}$; but this becomes noticeable only after a certain threshold value of $\mathrm{Gr}$ is exceeded (approximately $\mathrm{Gr}>10^{5}$ ). Figures $4 \mathrm{a}$ and $4 \mathrm{~b}$ show that the use of the more permeable porous blocks improves the heat transfer, more particularly with higher values of porous blocks thickness. Indeed, for $\mathrm{Gr}=10^{7}$ and $\mathrm{Da}=10^{-3}, \mathrm{Nu}_{\mathrm{b}}$ goes

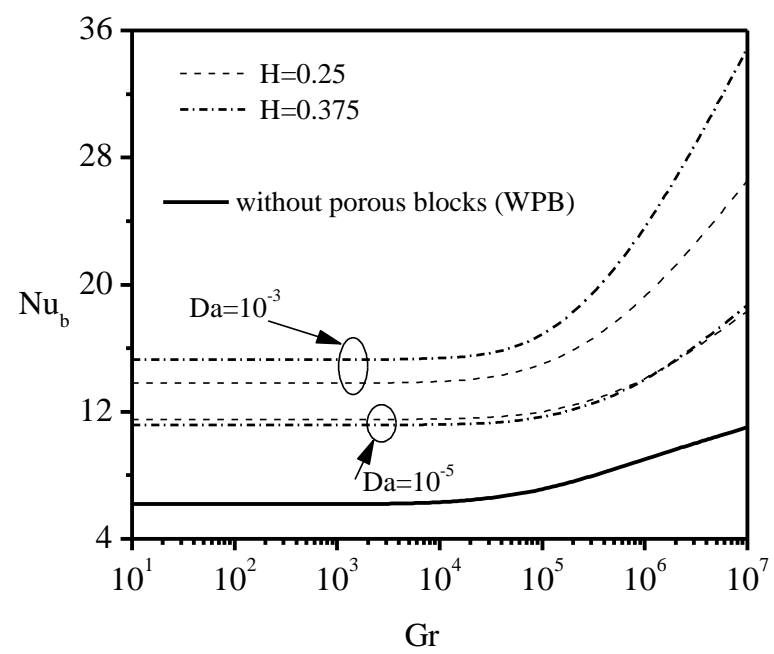

(a) $\mathrm{R}_{\mathrm{C}}=10$

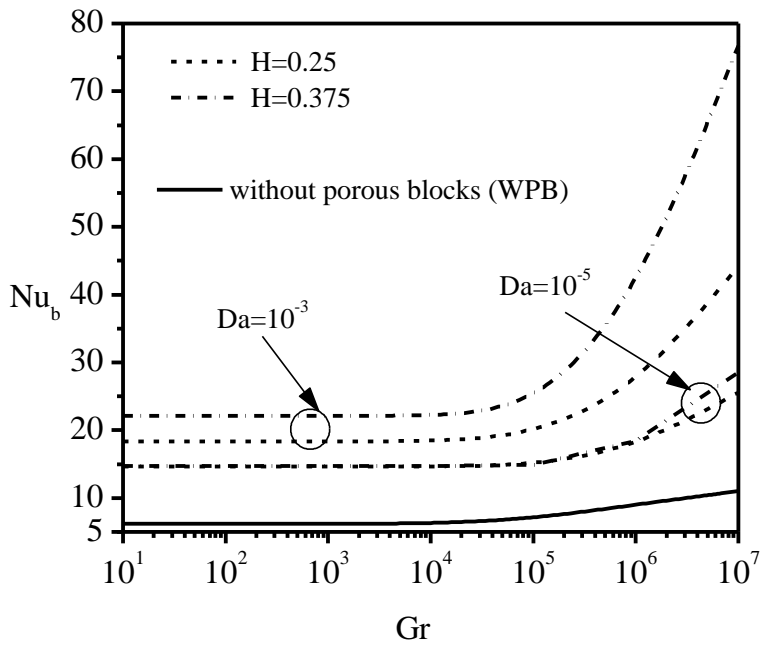

(b) $\mathrm{R}_{\mathrm{C}}=50$

Figure 4. Average Nusselt number

approximately from 45 for $\mathrm{H}=0.25$ to 75 for $\mathrm{H}=0.375$, i.e. an enhancement of $50 \%$. These enhancements are attributable to the combined effect of highly conductive porous blocks, buoyancy and the presence of much larger pore volume. Indeed, the porous blocks acting as extended surfaces for heat transfer.

\section{Friction factor}

The increased heat transfer in mixed convection and with presence of porous media does not come without a penalty. The pressure gradient required for a given mass flow rate through the duct also increases in magnitude.

The axial pressure drop for the flow can be expressed in terms of the friction factor $f$, where $f$ is given by the standard definition:

$$
f=\frac{-\left(\frac{\partial \mathrm{p}}{\partial \mathrm{z}}\right) \mathrm{d}}{\frac{1}{2} \rho \overline{\mathrm{w}}^{2}}
$$

If previous equation is rewritten in terms of the dimensionless variables, there results:

$$
f \mathrm{Re}=\frac{2}{\overline{\mathrm{W}}}
$$

with

$$
\operatorname{Re}=\frac{\rho \overline{\mathrm{w}} \mathrm{d}}{\mu}
$$

The pressure gradient and body force terms in the momentum equation for the radial direction are: 


$$
-\frac{\partial \mathrm{p}}{\partial \mathrm{r}}+\rho \mathrm{g} \cos \varphi
$$

The density $\rho$ is given by:

$$
\rho=\rho_{\mathrm{w}}[1-\beta(\mathrm{T}-\mathrm{Tw})]
$$

With a modified pressure $\mathrm{p}^{*}$ defined as:

$$
\mathrm{p}^{*}=\mathrm{p}-\rho_{\mathrm{W}} \mathrm{gr} \cos \varphi
$$

It is possible to write Eq. (14) as:

$$
-\frac{\partial \mathrm{p}}{\partial \mathrm{r}}+\rho \mathrm{g} \cos \varphi=-\frac{\partial \mathrm{p}^{*}}{\partial \mathrm{r}}-\rho_{\mathrm{w}} \mathrm{g} \beta\left(\mathrm{T}-\mathrm{T}_{\mathrm{w}}\right) \cos \varphi
$$

The corresponding terms in the $\varphi$ direction momentum equation can be similarly written as:

$$
-\frac{1}{\mathrm{r}} \frac{\partial \mathrm{p}}{\partial \varphi}-\rho \mathrm{g} \sin \varphi=-\frac{1}{\mathrm{r}} \frac{\partial \mathrm{p}^{*}}{\partial \varphi}+\rho_{\mathrm{w}} \mathrm{g} \beta\left(\mathrm{T}-\mathrm{T}_{\mathrm{w}}\right) \sin \varphi
$$

The dimensionless bulk temperature $\theta_{\mathrm{b}}$ is calculated from:

$$
\theta_{\mathrm{b}}=\frac{\iint \theta \mathrm{W} \eta \mathrm{d} \eta \mathrm{d} \varphi}{\iint \mathrm{W} \eta \mathrm{d} \eta \mathrm{d} \varphi}
$$

The dimensionless mean value of $\mathrm{W}$ is calculated from:

$$
\overline{\mathrm{W}}=\frac{\iint \mathrm{W} \eta \mathrm{d} \eta \mathrm{d} \varphi}{\iint \eta \mathrm{d} \eta \mathrm{d} \varphi}
$$

In the thermally developed region, the temperature $T_{w}$ and any local temperature $T$ of the fluid in the annulus vary linearly with $\mathrm{z}$ direction at the same constant rate determined by $\mathrm{Q}^{\prime}$, the rate of heat flow per unit axial length. The axial rate of rise of the fluid temperature $T$ can be related to the heat flow rate Q' by means of the overall heat balance.

$$
\frac{\partial \mathrm{T}}{\partial z}=\frac{\partial \mathrm{T}_{\mathrm{w}}}{\partial z}=\frac{\mathrm{Q}^{\prime}}{\rho \mathrm{C}_{\mathrm{P}} \overline{\mathrm{W}} \pi\left(\mathrm{R}_{\mathrm{O}}^{2}-\mathrm{R}_{\mathrm{i}}^{2}\right)}
$$

If $\overline{\mathrm{q}}$ represents the average heat flux at the surface of the inner cylinder, then $\overline{\mathrm{q}}$ and $\mathrm{Q}$ are related by:

$$
\mathrm{Q}^{\prime}=2 \pi \mathrm{R}_{\mathrm{i}} \overline{\mathrm{q}}
$$

Thus:

$$
\frac{\partial \mathrm{T}}{\partial z}=\frac{\partial \mathrm{T}_{\mathrm{w}}}{\partial z}=\frac{2 \overline{\mathrm{q}} \mathrm{R}_{\mathrm{i}}}{\rho \mathrm{C}_{\mathrm{P}} \overline{\mathrm{W}}\left(\mathrm{R}_{\mathrm{O}}^{2}-\mathrm{R}_{\mathrm{i}}^{2}\right)}
$$

The mean Nusselt number is defined as:

$$
\overline{\mathrm{h}}\left(\mathrm{T}_{\mathrm{w}}-\mathrm{T}_{\mathrm{b}}\right)=\overline{\mathrm{q}}
$$


Equation 24 is multiplied by $\left(\frac{\mathrm{d}}{\mathrm{k}_{\mathrm{f}}}\right) \times\left(\frac{1}{\mathrm{~T}_{\mathrm{w}}-\mathrm{T}_{\mathrm{b}}}\right)$, then we obtain:

Thus:

$$
\frac{\overline{\mathrm{h}} \mathrm{d}}{\mathrm{k}_{\mathrm{f}}}=\mathrm{Nu}_{\mathrm{b}}=\left(\frac{\overline{\mathrm{q}} \mathrm{d}}{\mathrm{k}_{\mathrm{f}}}\right) \times\left(\frac{1}{\mathrm{~T}_{\mathrm{w}}-\mathrm{T}_{\mathrm{b}}}\right)
$$

$$
\mathrm{Nu}_{\mathrm{b}}=-\frac{1}{\theta_{\mathrm{b}}}
$$

In the absence of the secondary flow, the product $f$ Re is constant, which depends only on the geometry and the permeability of the porous blocks. Figure 5 shows the product $f$ Re plotted as a function of Gr for different values of $\mathrm{H}$. The influence of buoyancy becomes noticeable only after a certain threshold value of Gr is exceeded, since Stronger secondary flows are associated with increasing values of $\mathrm{Gr}$. The increase in friction factor due to porous blocks is seen to be significantly large with lower values of Darcy number and with higher values of $H$. Indeed, the flow resistance increases with the Darcy number decrease and with the increase in the thickness of porous blocks.

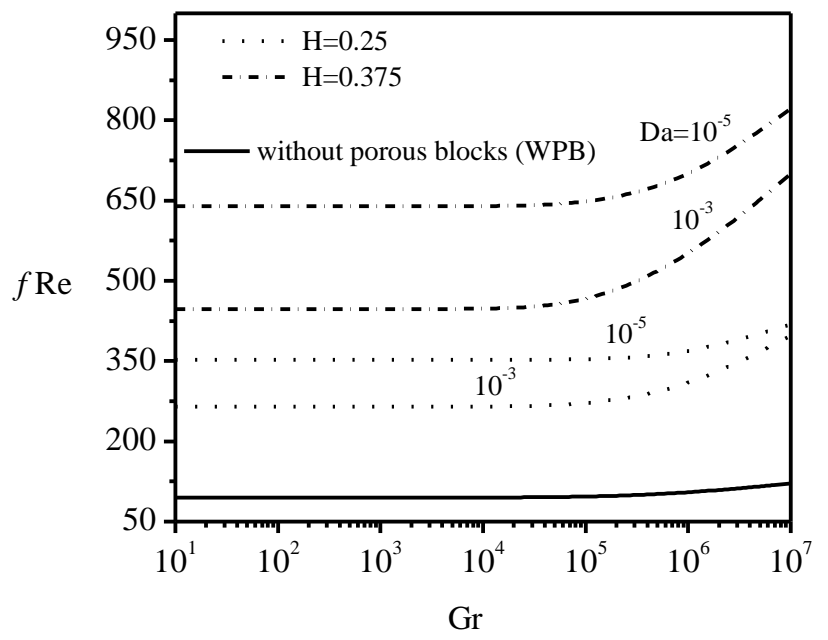

Figure 5. Friction factor for $\mathrm{R}_{\mathrm{C}}=10$

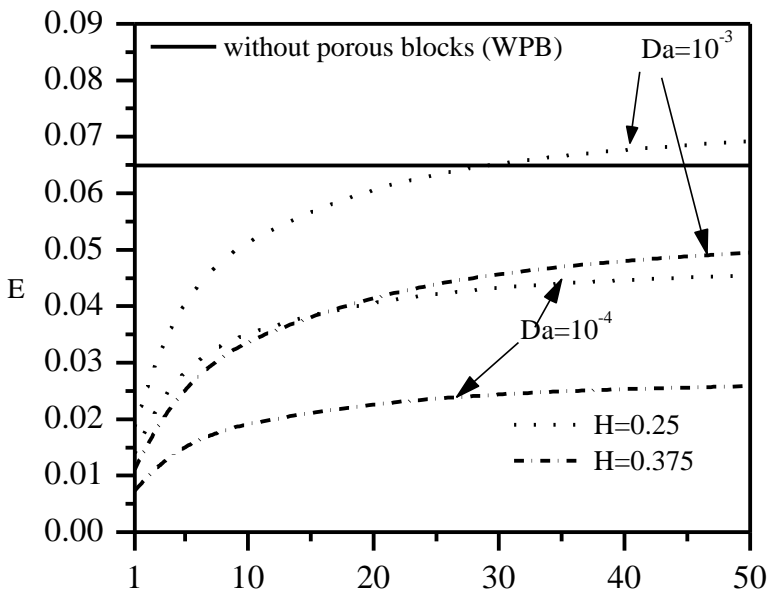

(a) ${ }^{\mathrm{R}} \mathrm{Gr}=10^{3}$

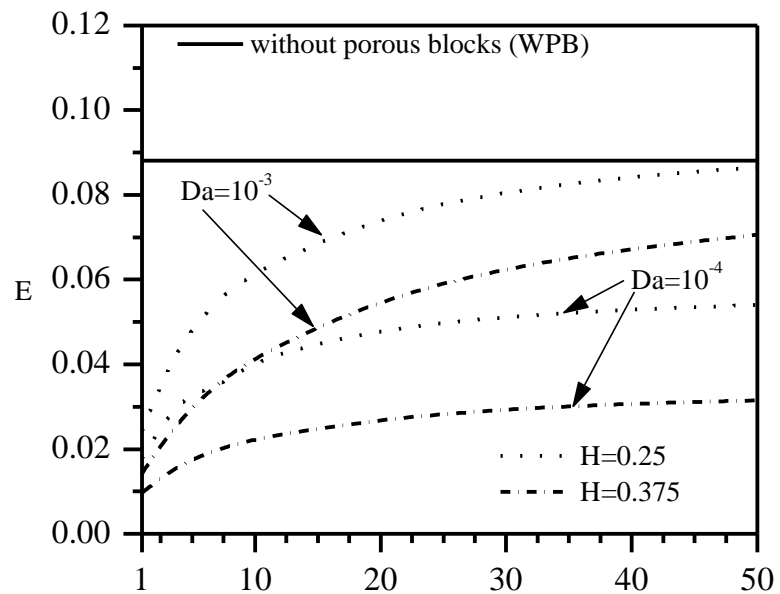

$\mathrm{R}_{\mathrm{d}}$ (b) $G r=10^{6}$

Figure 6. Ratio of mean Nusselt number to friction factor for 


\section{Ratio of mean Nusselt number to friction factor}

From the previous discussion of the overall heat transfer coefficient and the friction factor, it appears that $\mathrm{Nu}_{\mathrm{b}}$ increases with higher values of $\mathrm{H}$; and on the other hand $f$ Re increases with the presence of the porous matrix. For this antagonist effect a new parameter must be taken into consideration in design calculations. It corresponds to the ratio of the mean Nusselt number to the quantity defined as: . Figures $6 \mathrm{a}$ and $6 \mathrm{~b}$ show the ratio E plotted as a function of for different values of $\mathrm{H}$ and $\mathrm{Da}$. The plot for the situation without porous blocks is shown by solid line and is considered as a reference. It can be seen that the ratio $\mathrm{E}$ is significantly influenced by the Darcy number and the thickness of porous blocks. Thus, the combined effect of highly conductive porous blocks, the thickness $\mathrm{H}$ and the buoyancy is noticeable only for lower values of $\mathrm{Gr}$ and $\mathrm{Da}$ and only for $\mathrm{H}=0.25$. This enhancement occurs after a certain threshold value of is exceed (approximately).

\section{CONCLUSION}

According to the simulated results, the present research has led to the following conclusions:

It is found that the porous blocks significantly affect heat transfer and the fluid flow characteristics. In each half of the annulus divided by the vertical center line, the secondary flow generated by buoyancy, in the presence of the porous structure, has single, two, three or four eddies pattern depending on the thickness of porous blocks, Darcy number and Grashoff number.

The use of highly conductive porous blocks leads to a significant enhancement in heat transfer, particularly at $\mathrm{H}=0.375$; however the friction factor increases with.

The ratio of the average Nusselt number to friction factor is introduced to take account the challenge between increased heat transfer and decreased friction factor. The use of the porous structure, in the configuration proposed, is justified only when this parameter is greater than the values obtained for the fully fluid situation. This enhancement occurred for $\mathrm{Da}=10^{-3}$ and for $\mathrm{H}=0.25$ after certain threshold value of $\mathrm{R}_{\mathrm{C}}$ is exceeded.

\section{NOMENCLATURE}

$a \quad$ thermal diffusivity, $\mathrm{m}^{2} \cdot \mathrm{s}^{-1}$

$\mathrm{C}_{\mathrm{F}} \quad$ inertial coefficient

$\mathrm{C}_{\mathrm{P}} \quad$ specific heat at constant pressure, $\mathrm{J} . \mathrm{kg}^{-1} \cdot \mathrm{K}^{-1}$

$\mathrm{d} \quad$ hydraulic diameter, $\mathrm{d}=2\left(\mathrm{R}_{\mathrm{O}}-\mathrm{R}_{\mathrm{i}}\right)$

Da Darcy number, $\mathrm{Da}=\mathrm{K} / \mathrm{d}^{2}$

$\mathrm{E}$ ratio of mean Nusselt number to the product $(f \mathrm{Re}), \mathrm{E}=\left(\mathrm{Nu}_{\mathrm{b}} / f \mathrm{Re}\right)$

$f \quad$ Friction factor, Eq. (13)

$(f \mathrm{Re})_{0}$ value of $(f \mathrm{Re})$ in the absence of buoyancy

g gravitational acceleration

Gr Grashof number, $\mathrm{Gr}=\mathrm{g} \beta \overline{\mathrm{q}} \mathrm{d}^{4} /\left(v^{2} \mathrm{k}_{\mathrm{f}}\right)$

$\mathrm{h} \quad$ radial dimension of the each porous block, $\mathrm{m}$

$\mathrm{H}$ dimensionless radial dimension of each porous block, $\mathrm{H}=\mathrm{h} / \mathrm{d}$

$\mathrm{K}$ permeability of the porous blocks, $\mathrm{m}^{2}$

$\mathrm{k}_{\mathrm{f}} \quad$ thermal conductivity of the fluid, $\mathrm{W} \cdot \mathrm{m}^{-1} \cdot \mathrm{K}^{-1}$

$\mathrm{k}_{\mathrm{P}} \quad$ effective thermal conductivity of each porous

$\mathrm{Nu}$ local Nusselt number, Eq. (11)

$\mathrm{Nu}_{\mathrm{b}} \quad$ average Nusselt number, Eq. (12)

$\overline{\mathrm{Nu}_{0}} \quad$ average Nusselt number in the absence of buoyancy

$\mathrm{P}$ dimensionless pressure, $\mathrm{P}=\mathrm{p} * /\left(\rho(v / \mathrm{d})^{2}\right)$

$\mathrm{p} \quad$ pressure, $\mathrm{Pa}$

$\mathrm{p}^{*} \quad$ modified pressure, $\mathrm{p}^{*}=\mathrm{p}-\rho_{\mathrm{w}} \mathrm{gr} \cos \varphi$

$\operatorname{Pr} \quad$ Prandtl number, $\operatorname{Pr}=(v / a)_{\mathrm{f}}$

$\overline{\mathrm{q}} \quad$ average rate of heat transfer per unit area, W. $\mathrm{m}^{-2}$

Re Reynolds number, Eq. (15)

$\mathrm{R}_{\mathrm{i}} \quad$ radius of inner cylinder, $\mathrm{m}$

$\mathrm{R}_{\mathrm{o}} \quad$ radius of outer cylinder, $\mathrm{m}$ 
$\mathrm{RR}$ radius ratio, $\mathrm{RR}=\mathrm{R}_{\mathrm{o}} / \mathrm{R}_{\mathrm{i}}$

$\mathrm{R}_{\mathrm{v}} \quad$ viscosity ratio, $\mathrm{R}_{\mathrm{v}}=\mu_{\mathrm{e}} / \mu_{\mathrm{f}}$

$\mathrm{R}_{\mathrm{C}} \quad$ thermal conductivity ratio, $\mathrm{R}_{\mathrm{C}}=\mathrm{k} / \mathrm{k}_{\mathrm{f}}$

$\mathrm{r}$ radial coordinate, $\mathrm{m}$

$\mathrm{T} \quad$ temperature; ${ }^{\circ} \mathrm{C}$

$\mathrm{T}_{\mathrm{b}} \quad$ bulk temperature, ${ }^{\circ} \mathrm{C}$

$\mathrm{T}_{\mathrm{w}} \quad$ wall temperature of inner cylinder, ${ }^{\circ} \mathrm{C}$

$\mathrm{U} \quad$ dimensionless velocity in $\varphi$ coordinate, $\mathrm{U}=\mathrm{ud} / v$

$\mathrm{u} \quad$ velocity component in $\varphi$ coordinate, $\mathrm{m} \cdot \mathrm{s}^{-1}$

$\mathrm{V}$ dimensionless velocity in $\mathrm{r}$ coordinate, $\mathrm{V}=\mathrm{vd} / v$

$\mathrm{v} \quad$ velocity component in $\mathrm{r}$ coordinate, $\mathrm{m} \cdot \mathrm{s}^{-1}$

$\mathrm{W}$ dimensionless velocity in $\mathrm{z}$ direction,

$$
W=\frac{\mathrm{W}}{\left[\left(-\frac{\mathrm{dP}}{\mathrm{dz}}\right) \frac{\mathrm{d}^{2}}{\mu}\right]}
$$

$\mathrm{w} \quad$ velocity in $\mathrm{z}$ direction, $\mathrm{m} \cdot \mathrm{s}^{-1}$

$\overline{\mathrm{W}}$ mean value of $\mathrm{W}$

\section{REFERENCES}

[1] Huetz, J., \& Petit, J. P. (1974). Natural and Mixed Convection in Concentric Annular Spaces-Experimental and Theoretical Results for Liquid Metals. In 5th International Heat Transfer Conference, Tokyo (Vol. 3, pp. 169172).

[2] Nguyen, T. H., Vasseur, P., Robillard, L., \& Shekar, B. C. (1983). Combined free and forced convection of water between horizontal concentric cylinders. Journal of Heat Transfer, 105(3), 498-504.

[3] Hattori, N. (1979). Combined free and forced convection heat transfer for fully developed laminar flow in concentric annuli (Numerical Analysis). The Japan Society of Mechanical Engineers Transactions, 45, 227-239.

[4] Nieckele, A. O., \& Patankar, S. V. (1985). Laminar mixed convection in a concentric annulus with horizontal axis. Journal of heat transfer, 107(4), 902-909.

[5] Habib, M. A., \& Negm, A. A. A. (2001). Laminar mixed convection in horizontal concentric annuli with nonuniform circumferential heating. Heat and Mass Transfer/Waerme- Und Stoffuebertragung, 37(4-5), 427-435.

[6] Muralidhar, K. (1989). Mixed convection flow in a saturated porous annulus. International Journal of Heat and Mass Transfer, 32(5), 881-888.

[7] Nield, D. A., \& Bejan, A. (2013). Convection in porous media. Convection in Porous Media 3rd edition, Springer, New York, 2006.

[8] Vanover, D. E., \& Kulacki, F. A. (1987). Experimental Study of Mixed Convection in a Horizontal Porous Annulus. ASME Heat Transfer Division, 84, 61-66.

[9] Mohammed, H. A., Campo, A., \& Saidur, R. (2010). Experimental study of forced and free convective heat transfer in the thermal entry region of horizontal concentric annuli. International Communications in Heat and Mass Transfer, 37(7), 739-747.

[10] Ould-Amer, Y. (2010). Laminar mixed convection in horizontal concentric annuli with four porous blocks attached on the outside of the inner cylinder. ASME 10th Biennial Conference on Engineering Systems Design and Analysis (ESDA2010), July 12-14, 2010, Istanbul, Turkey, Paper no. ESDA2010-24077, 433-442.

[11] Venugopal, G., Balaji, C., \& Venkateshan, S. P. (2010). Experimental study of mixed convection heat transfer in a vertical duct filled with metallic porous structures. International Journal of Thermal Sciences, 49(2), 340-348.

[12] Ould-Amer Y. (2013), "Optimal heat transfer in horizontal concentric annuli with the presence of porous structure," International conference on mechanical engineering and technology, Amsterdam Nederland, 77, 486494.

[13] Ould-Amer Y. (2016). 3D Fully Developed Laminar Mixed Convection in Horizontal Concentric Annuli with the Presence of Porous Blocks. IACSIT International Journal of Engineering and Technology, Vol. 8, No. 2, 7682.

[14]Patankar, S. V. (1980). Numerical Heat Transfer and Fluid Flow. McGraw-Hill, New York, 1980. 